\title{
Chemical nonequilibrium effects on flow field for reusable launch vehicles
}

\author{
H. $\mathrm{Ma}^{1}$, L. Zhao ${ }^{1}$, and F.M. Wang ${ }^{1}$ \\ Institute of Mechanics, Chinese Academy of Sciences, 15 Beisihuanxi Road, Beijing, China
}

\begin{abstract}
High temperature chemical non-equilibrium phenomena have a great effect on the flow field around a reentry vehicle. A set of three dimensional Navier-Stokes equations have been solved by implicit finite volume NND scheme. Both ideal gas viscous flow and chemical non-equilibrium flow are calculated for a spherical-cone at a small angle of attack. The results of the two flows have been compared and the effect of chemical non-equilibrium has been analyzed. The effect of wall material's properties, such as catalysis and radiation were studied. The results are in good agreement with the referenced paper.
\end{abstract}

\section{Introduce}

Today numerical techniques are able to calculate the entry heating during the reentry process. Flight during this process is known to be characterized by nonequilibrium flow in the shock layer. In the 80's of the last century, Shinn's research [1] shows that when a nonequilibrium dissociative reacting code was adapted to shuttle's flow field, the result is quite far from that predicted by assuming chemical equilibrium. The predicted convective heating was in good agreement with the STS-2 flight data. That shows the effect of nonequilibrium is very important for a vehicles' thermal protecting system (TPS)'s design. Because the reusable vehicle's main heating dissipation method is radiation, the TPS's material's property is very important for a reusable vehicle, and the prediction of their surface heating is a key procedure in the design of TPS.

There are two problems in the simulation of high temperature chemical nonequilibrium flow. First, there should have enough grids in the range of $y^{+}<30$ near the wall that ensures the resolution in the simulation of three-dimension flows. But the density of grid is requiring greater computer resources; Second, the chemical stiffness produces unstable calculation and convergence is difficult to obtain, especially in three-dimension computation. o resolve these problems, we use a hybrid integration to calculate viscous items according to the definition of gradient and divergence. We use implicit fully-coupled method to calculate the chemical resource item. To ensure stability, we take two stability criteria to determine the calculation time step.

This paper studied the effect of chemical nonequilibrium in the shock layer and the wall material's equilibrium radiation's effect to reentry vehicle's flowfield during a hypersonic reentry. We selected a blunt spherical cone with the same nose radius with X-33 and a 10 half cone angle. A set of three dimensional Navier-Stokes equations were solved by implicit finite volume NND scheme. The results are in good agreement with referenced paper. The differences between perfect gas results and chemical nonequilibrium gas results and the effect of surface catalysis and material's radiation character are examined. 


\section{Governing equations}

The integral three-dimensional conservative N-S equations is

$$
\begin{aligned}
& \int_{\Omega} \frac{\partial}{\partial t} \rho_{i} d \Omega+\oint_{\Gamma} \boldsymbol{V} \cdot d \boldsymbol{S}=\oint_{\Gamma} \rho_{i} D_{i} \nabla y_{i} \cdot d \boldsymbol{S}+\int_{\Omega} \dot{\omega}_{i} d \Omega \\
& \int_{\Omega} \frac{\partial}{\partial t}(\rho \boldsymbol{V}) d \Omega+\oint_{\Gamma} \rho \boldsymbol{V}(\boldsymbol{V} \cdot d \boldsymbol{S})=\oint_{\Gamma} \tau_{n} \cdot d \boldsymbol{S} \\
& \int_{\Omega} \frac{\partial}{\partial t} \rho E d \Omega+\oint_{\Gamma} \rho E \boldsymbol{V} \cdot d \boldsymbol{S}=\oint_{\Gamma} \boldsymbol{\tau}_{n} \cdot \boldsymbol{q} d \boldsymbol{S}+\oint_{\Gamma} k \nabla T \cdot d \boldsymbol{S}+\oint_{\Gamma} \rho \sum_{i=1}^{n s} h_{i} D_{i} \nabla y_{i} \cdot d \boldsymbol{S}
\end{aligned}
$$

in which $\boldsymbol{V}=u \boldsymbol{i}+v j+w \boldsymbol{k}, \dot{\omega}$ is chemical source term, $D_{i}$ is diffusion coefficient, $y_{i}$ is mass fraction of species $i$.

Since we are concerned with the aerodynamic heating during reentry, we omitted the ionization character. We use a five species chemical reaction model, and the Dun and Kang's model to calculate the reaction rates. The thermodynamic properties of each species are extended to higher temperatures with the same method of polynomial curve fit applied to the tabulated data of Browne. The various transport properties are calculated with Wilkes' semiempirical mixing rule with curve fitting for species viscosities given by Blottner et al, and a constant Schmidt number with Fick's law for the mass diffusion [2].

\section{Numerical method}

We use implicit finite volume NND scheme for solving [3] the viscous N-S equations. For the viscous terms, we use integration to calculate according to the definition of gradient and divergence.

$$
\operatorname{grad} \Phi \approx \frac{1}{V}\left[\sum_{l=1}^{6} \Phi_{l} S_{l x} i+\sum_{l=1}^{6} \Phi_{l} S_{l y} j+\sum_{l=1}^{6} \Phi_{l} S_{l z} k\right]
$$

$\Phi$ is a scalar, $\Phi_{l}$ is component of $\Phi$ normal to a computational cell face $l, S_{l}$ is face $l$ 's area vector, $V$ is cell's volume. This method has good conservation property and then improve the computation efficiency .

The pressure term and surface heating flux can be expressed by

$$
\begin{aligned}
& \hat{p}=p+\frac{2}{3 V} \mu\left(\sum_{l=1}^{6} u_{l} S_{l x}+\sum_{l=1}^{6} v_{l} S_{l y}+\sum_{l=1}^{6} w_{l} S_{l z}\right) \\
& \left.q_{n} \cdot \Delta S\right|_{w}=-\left(\oint_{\Gamma} k \nabla T \cdot d \boldsymbol{S}+\oint_{\Gamma} \rho \sum_{i=1}^{n s} h_{i} D_{i} \nabla y_{i} \cdot d \boldsymbol{S}\right)
\end{aligned}
$$

There are great difference between the flow characteristic time and chemical reaction characteristic time. The source term actually include a chemical relaxation time $\tau_{\text {chem }}=\left(\frac{\partial \dot{\omega}_{i}}{\partial \rho_{i}}\right)^{-1}$, but the flow's macroscopic time scale is $\tau_{f l o w}=\frac{\Delta x}{u+a}$, so there are two time stability critera in chemical nonequilibrium flow,

(1) For nonequilibrium relaxation process, time step $\Delta t$ should be smaller than the fastest chemical reaction, $\Delta t<B \tau_{\text {chem }}$;

(2) According to stability criterion of hyperbolic equation, the time step should satisfy $\Delta t<k \tau_{\text {flow }}$.In which, $k$ and $B$ are empirical parameters determined by calculation scheme and calculation conditions and $a$ is sound speed.

When $\tau_{\text {chem }} \ll \tau_{\text {flow }}$, the stiffness problem will arise, the general method is introduced through a time precondition matrix. At the same time, according to the above two critera, we take time step as $\Delta t<k_{1} \tau_{f l o w}$, in which $k_{1}$ is a parameter determined by the chemical characteristic time in the flow in the range1.0 $\times 10^{-2}<k_{1}<1.0$. 


\section{Boundary condition}

At the wall, we take no slip, no temperature jump,no pressure gradient condition. For species concentration, both the equilibrium catalytic(surface catalytic efficiency $\gamma=1$ )and the no catalytic wall(surface catalytic efficiency $\gamma=0$ ) cases are calculated. Finally we solve energy balanced equations on the surface to examine the material's radiation influence, assuming a radiative equilibrium wall condition.

$$
k \frac{\partial T}{\partial n}+\sum_{s=1}^{n s}\left(\rho D_{s} h_{s} \frac{\partial Y_{i}}{\partial \eta}\right)=\epsilon(T) \sigma T_{w}^{4}
$$

\section{Results}

We take the trajectory of reusable vehicle as the free stream condition [4] and calculate the perfect gas flow and chemical nonequilibrium flow respectively. The altitude is $67.4 \mathrm{~km}$. Free stream Mach number is $\mathrm{Ma}=11.7$. Attack angle is 11.75 . Table 1 is the comparison of the calculated result and referenced result. The nonequilibrium result is lower than the perfect gas result, since the chemical reactions behind the shock are heat absorbing reactions, causing a drop in the temperature behind the shock, and thence a lower surface heat flux. The nonequilibrium chemical reaction causes a decrease in the shock distance, resulting the nonequilibrium stagnation pressure little higher than the perfect gas result in table 1.

Table 1. Comparison of stagnation heat flux and pressure

\begin{tabular}{llll}
\hline & perfect gas & nonequilibrium gas & ref. \\
\hline heat flux $Q\left(M W / m^{2}\right)$ & 0.142 & 0.0763 & 0.06 \\
pressure $P(P a)$ & 921.95 & 1079.16 & - \\
\hline
\end{tabular}

Fig. 1 shows the comparison of temperature isolines of chemical nonequilibrium flow and perfect gas flow. We see an obvious temperature drop in nonequilibrium chemical flow. Fig. 2 shows the distribution of each species' mass fraction along the stagnation line. We see that the oxygen molecule is dissociated completely, but the dissociation of nitrogen molecules is small, the air behind the shock is mainly composed by $N_{2}, N, O$. Fig. 3 shows the surface heat flux's comparison along the body. We see that the heat flux of nonequilibrium flow is much lower than the case for perfect gas.

To examine the influence of surface catalysis and the wall material's radiation performance, we selected 4 trajectory point condition of reentry vehicle X-33. Table 2 shows the trajectory conditions.

Fig. 4 shows surface catalysis' effect to wall's heat flux. ECW represents equilibrium catalysis wall and NCW the non-catalysis wall. The differences between ECW and NCW results may represent the degree of nonequilibrium in the flow field. This suggests that the nonequilibrium is most serious at the point of maximum heat, at the same time the wall catalysis effect on heat flux is greatest. Fig. 5 shows the wall temperature's distribution along the body. We can see the highest wall temperature appeared at the peak of heating. The stagnation wall temperature reaches to $1304 \mathrm{~K}$ near the TPS's maximum temperature. 

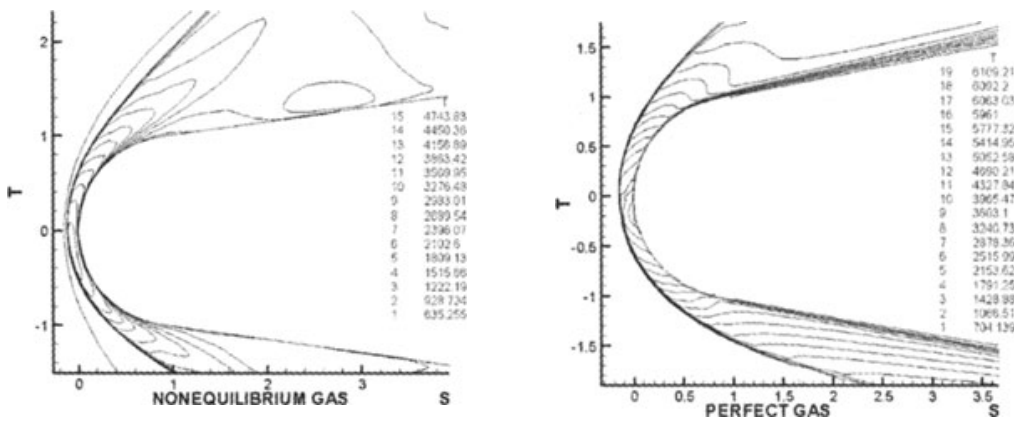

Fig. 1. Comparison of nonequilibrium flow and perfect gas flow temperature isoline, $67.4 \mathrm{~km}$, $\mathrm{Ma}=11.7$

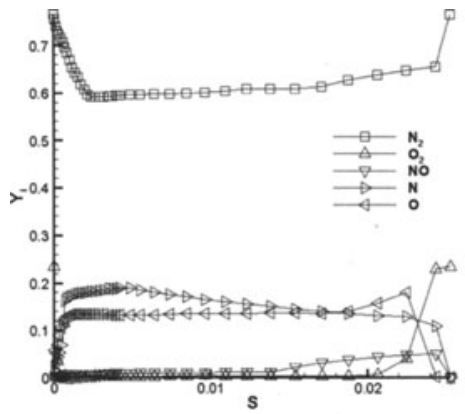

Fig. 2. Species mass fraction along stagnation line, $67.4 \mathrm{~km}, \mathrm{Ma}=11.7$

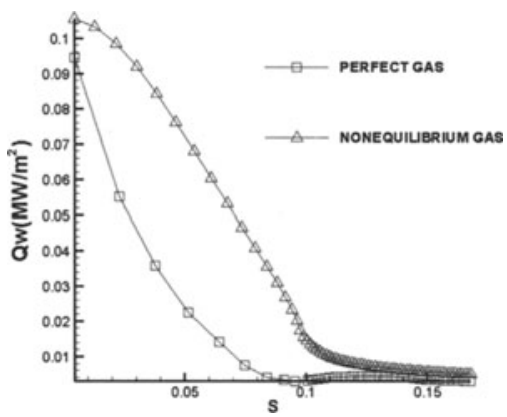

Fig. 3. Surface heat flux's comparison along the body, $67.4 \mathrm{~km}, \mathrm{Ma}=11.7$ 
Table 2. X-33 Trajectory Point

\begin{tabular}{llllll}
\hline & Alt. km & $V_{\infty} \mathrm{km} / \mathrm{s}$ & Den. kg/m & $T_{\infty} \mathrm{K}$ & $\mathrm{Ma}$ \\
\hline 1 & 67.4 & 3.3717 & $1.18 \times 10^{-4}$ & 266.6 & 11.17 \\
2 & 62.3 & 4.579 & $2.324 \times 10^{-4}$ & 240.8 & 14.72 \\
3 & 58.5 & 4.2962 & $3.74 \times 10^{-4}$ & 251 & 13.53 \\
4 & 57.7 & 3.7786 & $4.092 \times 10^{-4}$ & 253.3 & 11.84 \\
\hline
\end{tabular}

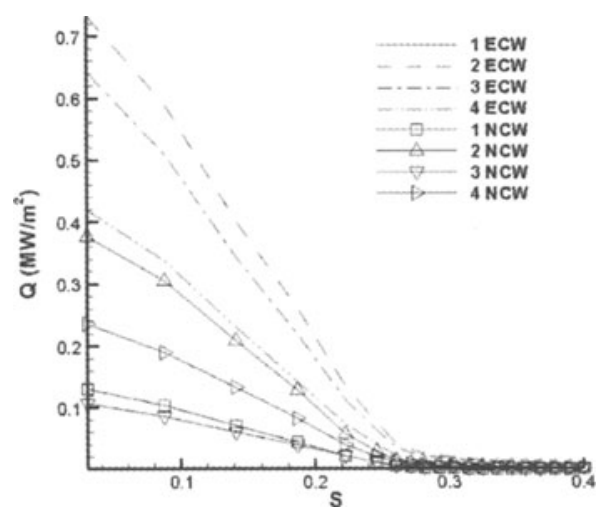

Fig. 4. The effect of catalysis to wall heat flux.

\section{Conclusion}

This paper study the nonequilibrium chemical flow field and surface properties' effects on a blunt body. The results are calculated using an implicit finite volume method that proved to be success in predicting nonequilibrium heating. The following conclusions are obtained: (1) On the X-33 trajectory, there are great differences in the nonequilibrium chemical result and the preface gas result. But the effect due to a drop in the temperature and heat flux on the pressure is small. (2) At the point of maximum heat, the flow field is of higher degree of nonequilibrium with substantial nitrogen dissociation. The wall material's catalytic property will significantly affect the heat flux at the wall. Hence, a selection of a TPS surface with small recombination rate will be more beneficial. (3) The wall's radiation effect is significant. So we should select the TPS's material with large emissivity coefficient to reduce the TPS's weight and avoid the damage due to aerodynamic heating.

\section{References}

1. J. L. Shinn: Chemical nonequilibrium effects on flowfields for aeroassisted orbital transfer vehicles. J. spacecraft and rockets A 1, 22 (1985)

2. P. A. Gnoffo,R. N. Gupta, J.L. Shinn: An upwind-based point-implicit relaxation algorithm for viscous compressible perfect-gas flows. NASA TP-2953,1990 


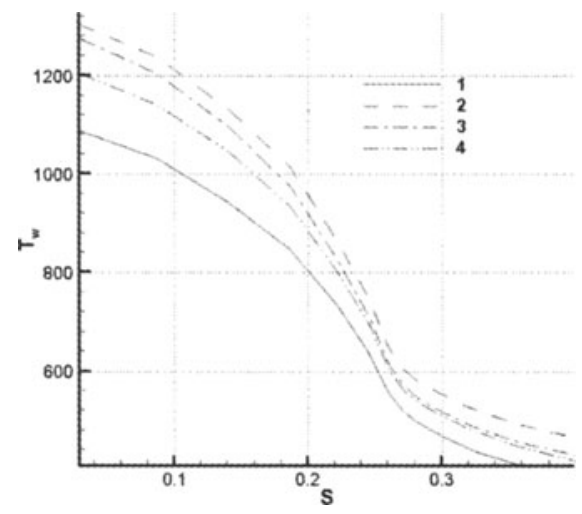

Fig. 5. The effect of radiation to wall temperature

3. R. F. Chen: Numerical Simulation of Invisid Steady Hypersonic Flow around Three Dimensional Body. Doctor Thesis, Beijing University, China

4. D. Olynick: Trajectory-based thermal protectionsystem sizing for an X-33 winged behicle concept. J. spacecraft and rockets A 3, 35 (1998) 